\title{
Seismic wave extrapolation in isotropic and anisotropic media using lowrank symbol approximation
}

\author{
Sergey Fomel, Lexing Ying, and Xiaolei Song, (The University of Texas at Austin)
}

\begin{abstract}
We consider the problem of constructing a wave extrapolation operator in a variable and possibly anisotropic medium. Our construction involves Fourier transforms in space combined with the help of a lowrank approximation of the space-wavenumber wave-propagator matrix. A lowrank approximation implies selecting a small set of representative spatial locations and a small set of representative wavenumbers. We present a mathematical derivation of this method, a description of the lowrank approximation algorithm, and numerical examples which confirm the validity of the proposed approach. Wave extrapolation using lowrank approximation can be applied to seismic imaging by reverse-time migration in $3 \mathrm{D}$ heterogeneous isotropic or anisotropic media.
\end{abstract}

\section{Introduction}

Wave extrapolation in time plays an important role in seismic imaging (reverse-time migration), modeling, and full waveform inversion. Conventionally, extrapolation in time is performed by finite-difference methods (Etgen 1986). Spectral methods (Tal-Ezer et al. 1987; Reshef et al. 1988) have started to gain attention recently and to become feasible thanks to the increase in computing power. The attraction of spectral methods is in their superb accuracy and, in particular, in their ability to suppress dispersion artifacts (Etgen and Brandsberg-Dahl 2009).

Theoretically, the problem of wave extrapolation in time can be reduced to analyzing numerical approximations to the mixed-domain space-wavenumber operator (Wards et al. 2008). In this paper, we propose a systematic approach to designing wave extrapolation operators by approximating the space-wavenumber matrix symbol with a lowrank decomposition. A lowrank approximation implies selecting a small set of representative spatial locations and a small set of representative wavenumbers. The optimized separable approximation or OSA (Song 2001) was previously employed for wave extrapolation (Zhang and Zhang 2009; Du et al. 2010) and can be considered as another form of lowrank decomposition. However, the decomposition algorithm in OSA is significantly more expensive, especially for anisotropic wave propagation, because it involves eigenfunctions rather than rows and columns of the original extrapolation matrix. Our algorithm can also be regarded as an extension of the interpolation algorithm of Etgen and Brandsberg-Dahl (2009), with optimally selected reference velocities and weights. Another related method is the Fourier finite-difference method proposed by Song and Fomel (2010), which requires only one pair of forward and inverse Fourier transforms per time step. 